\title{
Some identities involving the Dirichlet $L$-function
}

by

\section{Zhefeng Xu and Wenpeng Zhang (Xi'an)}

1. Introduction. Let $q \geq 3$ be an integer, $\chi$ be a Dirichlet character modulo $q$ and

$$
((x))= \begin{cases}x-[x]-1 / 2 & \text { if } x \text { is not an integer, } \\ 0 & \text { if } x \text { is an integer. }\end{cases}
$$

If $\chi$ is a primitive character, $\mathrm{H}$. Walum [3] established a connection between $((x))$ and the Dirichlet $L$-function $L(s, \chi)$ as follows:

$$
\sum_{a=1}^{q} \chi(a)\left(\left(\frac{a}{q}\right)\right)= \begin{cases}-\frac{\tau(\chi)}{\pi i} L(1, \bar{\chi}) & \text { if } \chi(-1)=-1, \\ 0 & \text { if } \chi(-1)=1,\end{cases}
$$

where

$$
\tau(\chi)=\sum_{a=1}^{q} \chi(a) e\left(\frac{a}{q}\right)
$$

is the Gauss sum, and $e(y)=e^{2 \pi i y}$. By using this connection he obtained the beautiful exact formula for the mean value of $L(1, \chi)$ in the case of $q=p$ a prime:

$$
\sum_{\substack{\chi \bmod p \\ \chi(-1)=-1}}|L(1, \chi)|^{2}=\frac{(p-1)^{2}(p-2)}{12 p^{2}} \pi^{2} .
$$

For general $q$, the second author [5] got the following identity:

$$
\sum_{\substack{\chi \bmod q \\ \chi(-1)=-1}}|L(1, \chi)|^{2}=\frac{\pi^{2}}{12} \cdot \frac{\phi^{2}(q)}{q^{2}}\left(q \prod_{p \mid q}\left(1+\frac{1}{p}\right)-3\right),
$$

where $\phi(q)$ is the Euler function. In [6], another proof of (1) was given by the second author. This new method can also be used to calculate the general 
mean value

$$
\sum_{\substack{\chi \bmod q \\ \chi(-1)=(-1)^{n}}} L(n, \chi) L(m, \bar{\chi})
$$

if $2 \mid(n+m)$, where $n, m$ are positive integers (see [2]). But for the case $2 \nmid(n+m)$, this method does not work even for $q=p$ and $m=1$.

The present work deals mainly with some mean values involving the Dirichlet $L$-function by using the arithmetical properties of the character sums over short intervals and the periodic Bernoulli polynomials $\bar{B}_{n}(x)$, where $\bar{B}_{n}(x)=B_{n}(x-[x]),[x]$ denotes the greatest integer less than or equal to $x$, and $B_{n}(x)$ are the classical Bernoulli polynomials defined by the following exponential generating function:

$$
\frac{t e^{x t}}{e^{t}-1}=\sum_{n=0}^{\infty} B_{n}(x) \frac{t^{n}}{n !}
$$

Finally, some new connections between the Dirichlet $L$-function and the periodic Bernoulli polynomials are obtained. Namely, we shall prove the following results:

TheOREM 1. Let $p \geq 5$ be a prime and $n$ be a positive integer. Then we have the identities

$$
\begin{aligned}
& \sum_{\substack{\chi \bmod p \\
\chi(-1)=1}} L\left(1, \bar{\chi} \chi_{4}\right) L(n, \chi) \\
& \quad=-\frac{\pi \chi_{4}(p)(p-1)}{p}\left[\frac{\zeta(n)}{p^{n}}\left[\frac{p}{4}\right]+\frac{2^{n-1}(-1)^{n / 2} \pi^{n}}{n !} \sum_{r \leq[p / 4]} \bar{B}_{n}\left(\frac{r}{p}\right)\right]
\end{aligned}
$$

if $n$ is even, and

$$
\sum_{\substack{\chi \bmod p \\ \chi(-1)=-1}}(2+\bar{\chi}(2)-\bar{\chi}(4)) L(1, \bar{\chi}) L(n, \chi)=\frac{(2 i \pi)^{n+1}(p-1)}{2 n ! p} \sum_{r \leq[p / 4]} \bar{B}_{n}\left(\frac{r}{p}\right)
$$

if $n$ is odd, where $\chi_{4}$ denotes the primitive character modulo 4 and $\zeta(n)$ the Riemann zeta function.

TheORem 2. Let $p \geq 3$ be a prime and $n$ be a positive integer. Then we have the identities

$$
\begin{aligned}
\sum_{\substack{\chi \bmod p \\
\chi(-1)=1}}(\bar{\chi}(2)-2) L(1, \bar{\chi}) L(n, \chi) \\
\quad=-\frac{\pi(p-1)}{2 i p}\left[\frac{\zeta(n)(p-1)}{p^{n}}+\frac{2^{n}(-1)^{n / 2} \pi^{n}}{n !} \sum_{r \leq(p-1) / 2} \bar{B}_{n}\left(\frac{r}{p}\right)\right]
\end{aligned}
$$


if $n$ is even, and

$$
\sum_{\substack{\chi \bmod p \\ \chi(-1)=-1}}(\bar{\chi}(2)-2) L(1, \bar{\chi}) L(n, \chi)=\frac{(2 i)^{n} \pi^{n+1}(p-1)}{2 i n ! p} \sum_{r \leq(p-1) / 2} \bar{B}_{n}\left(\frac{r}{p}\right)
$$

if $n$ is odd.

In our theorems, the terms

$$
\sum_{r \leq[p / 4]} \bar{B}_{n}\left(\frac{r}{p}\right) \text { and } \sum_{r \leq(p-1) / 2} \bar{B}_{n}\left(\frac{r}{p}\right)
$$

could be easily calculated by using the explicit expression of the Bernoulli polynomials. In particular, for $n=1,2,3$, noting that

$$
B_{1}(x)=x-\frac{1}{2}, \quad B_{2}(x)=x^{2}-x+\frac{1}{6}, \quad B_{3}(x)=x^{3}-\frac{3}{2} x^{2}+\frac{1}{2} x,
$$

we immediately get the following exact formulae:

Corollary 1. Let $p \geq 5$ be a prime. Then we have the identities

$$
\sum_{\substack{\chi \bmod p \\ \chi(-1)=1}} L\left(1, \bar{\chi} \chi_{4}\right) L(2, \chi)
$$

$$
= \begin{cases}\frac{\pi^{3}(p-1)^{2}}{64 p}\left(1-\frac{14}{3 p}-\frac{5}{3 p^{2}}\right) & \text { if } p \equiv 1(\bmod 4), \\ -\frac{\pi^{3}(p-3)}{64}\left(1-\frac{3}{p}-\frac{1}{p^{2}}+\frac{3}{p^{3}}\right) & \text { if } p \equiv 3(\bmod 4),\end{cases}
$$

$$
\begin{aligned}
& \sum_{\substack{\chi \bmod p \\
\chi(-1)=-1}}(2+\bar{\chi}(2)-\bar{\chi}(4))|L(1, \chi)|^{2} \\
& = \begin{cases}\frac{3 \pi^{2}(p-1)}{16}\left(1-\frac{1}{p}\right)^{2} & \text { if } p \equiv 1(\bmod 4), \\
\frac{3 \pi^{2}(p-3)}{16}\left(1-\frac{4}{3 p}+\frac{1}{3 p^{2}}\right) & \text { if } p \equiv 3(\bmod 4)\end{cases}
\end{aligned}
$$

and

$$
\begin{aligned}
& \sum_{\substack{\chi \bmod p \\
\chi(-1)=-1}}(2+\bar{\chi}(2)-\bar{\chi}(4)) L(1, \bar{\chi}) L(3, \chi) \\
& = \begin{cases}\frac{3 \pi^{4}(p+3)(p-1)^{2}}{256 p^{2}}\left(1-\frac{2}{3 p}-\frac{1}{3 p^{2}}\right) & \text { if } p \equiv 1(\bmod 4), \\
\frac{3 \pi^{4}(p-3)}{256}\left(1+\frac{2}{3 p}-\frac{4}{3 p^{2}}-\frac{2}{3 p^{3}}+\frac{1}{3 p^{4}}\right) & \text { if } p \equiv 3(\bmod 4) .\end{cases}
\end{aligned}
$$


Corollary 2. Let $p \geq 3$ be a prime. Then we have the identities

$$
\begin{aligned}
\sum_{\substack{\chi \bmod p \\
\chi(-1)=1}}(\bar{\chi}(2)-2) L(1, \bar{\chi}) L(2, \chi) & =\frac{i \pi^{3}}{12}\left(1-\frac{1}{p}-\frac{1}{p^{2}}+\frac{1}{p^{3}}\right), \\
\sum_{\substack{\chi \bmod p \\
\chi(-1)=-1}}(\bar{\chi}(2)-2)|L(1, \chi)|^{2} & =-\frac{\pi^{2} p}{8}\left(1-\frac{1}{p}\right)^{3}, \\
\sum_{\substack{\chi \bmod p \\
\chi(-1)=-1}}(\bar{\chi}(2)-2) L(1, \bar{\chi}) L(3, \chi) & =-\frac{\pi^{4}(p-1)^{3}(p+1)^{2}}{96 p^{4}} .
\end{aligned}
$$

2. Some lemmas. To prove the theorems, we need some lemmas.

LEMMA 1. Let $\chi$ be a primitive character modulo $m$ with $\chi(-1)=-1$. Then

$$
\frac{1}{m} \sum_{b=1}^{m} b \chi(b)=\frac{i}{\pi} \tau(\chi) L(1, \bar{\chi}) .
$$

Proof. This can be easily deduced from Theorems 12.11 and 12.20 of [1].

LEMMA 2 ([4, Lemma 2]). Let $q \geq 5$ be an odd integer and $\chi$ be a primitive Dirichlet character modulo $q$ such that $\chi(-1)=1$. Then

$$
\sum_{a=1}^{[q / 4]} \chi(a)=-\frac{i \bar{\chi}(4)}{2 \pi} \tau\left(\chi \chi_{4}\right) L\left(1, \bar{\chi} \chi_{4}\right)
$$

where $\chi_{4}$ is the primitive Dirichlet character modulo 4.

LEMma 3. Let $q \geq 3$ be an odd integer. For any nonprincipal character $\chi \bmod q$,

$$
\sum_{a=1}^{q} a \chi(a)=\frac{\chi(2) q}{1-2 \chi(2)} \sum_{a=1}^{(q-1) / 2} \chi(a)
$$

Proof. From the properties of Dirichlet characters, we have

$$
\begin{aligned}
\sum_{a=1}^{q} 2 a & \chi(2 a) \\
& =\sum_{a=1}^{(q-1) / 2} 2 a \chi(2 a)+\sum_{a=(q+1) / 2}^{q} 2 a \chi(2 a) \\
& =\sum_{a=1}^{(q-1) / 2} 2 a \chi(2 a)+\sum_{a=1}^{(q+1) / 2}(2 a-1) \chi(q+2 a-1)+q \sum_{a=1}^{(q+1) / 2} \chi(2 a-1) \\
& =\sum_{a=1}^{q} a \chi(a)+q \sum_{a=1}^{(q+1) / 2} \chi(2 a-1) .
\end{aligned}
$$


Noting that

we can write

$$
\sum_{a=1}^{(q+1) / 2} \chi(2 a-1)+\sum_{a=1}^{(q-1) / 2} \chi(2 a)=\sum_{a=1}^{q} \chi(a)=0
$$

$$
\begin{aligned}
(1-2 \chi(2)) \sum_{a=1}^{q} a \chi(a) & =\sum_{a=1}^{q} a \chi(a)-\sum_{a=1}^{q} 2 a \chi(2 a)=q \sum_{a=1}^{(q-1) / 2} \chi(2 a) \\
& =\chi(2) q \sum_{a=1}^{(q-1) / 2} \chi(a),
\end{aligned}
$$

as desired.

LEMma 4. Let $q \geq 3$ be an odd integer and $\chi$ be a primitive Dirichlet character modulo $q$ such that $\chi(-1)=-1$. Then

$$
\sum_{a=1}^{[q / 4]} \chi(a)=\frac{2+\bar{\chi}(2)-\bar{\chi}(4)}{2 i \pi} \tau(\chi) L(1, \bar{\chi}) .
$$

Proof. We consider two cases. First, we suppose $q \equiv 1(\bmod 4)$. From the properties of the Dirichlet character modulo $q$, we can write

$$
\begin{aligned}
4 \chi(4) & \sum_{a=1}^{q-1} a \chi(a) \\
= & \sum_{a=1}^{(q-1) / 4} 4 a \chi(4 a)+\sum_{a=(q+3) / 4}^{(2 q-2) / 4} 4 a \chi(4 a) \\
& +\sum_{a=(2 q+2) / 4} 4 a \chi(4 a)+\sum_{a=(3 q+1) / 4}^{(3 q-3) / 4} 4 a \chi(4 a) \\
= & \sum_{a=1}^{(q-1) / 4} 4 a \chi(4 a)+\sum_{a=1}^{(q-1) / 4}(4 a+q-1) \chi(4 a-1) \\
& +\sum_{a=1}^{(q-1) / 4}(4 a+2 q-2) \chi(4 a-2)+\sum_{a=1}^{(q-1) / 4}(4 a+3 q-3) \chi(4 a-3) \\
= & \sum_{a=1}^{q-1} a \chi(a)+\chi(4) q \sum_{a=1}^{(q-1) / 4} \chi(a-\overline{4}) \\
& +2 \chi(4) q \sum_{a=1}^{(q-1) / 4} \chi(a-2 \cdot \overline{4})+3 \chi(4) q \sum_{a=1} \chi(a-3) .
\end{aligned}
$$


Note that $\overline{4} \equiv(3 q+1) / 4(\bmod q)$ if $q \equiv 1(\bmod 4)$. So from $(2)$, we have

$$
\begin{aligned}
4 \chi(4) \sum_{a=1}^{q-1} a \chi(a) & \\
= & \sum_{a=1}^{q-1} a \chi(a)-\chi(4) q \sum_{a=(2 q+2) / 4}^{(3 q-3) / 4} \chi(a) \\
& -2 \chi(4) q \sum_{a=(q+3) / 4}^{(2 q-2) / 4} \chi(a)-3 \chi(4) q \sum_{a=1}^{(q-1) / 4} \chi(a) \\
= & \sum_{a=1}^{q-1} a \chi(a)-\chi(4) q \sum_{a=(q+3) / 4}^{(2 q-2) / 4} \chi(a)-3 \chi(4) q \sum_{a=1}^{(q-1) / 4} \chi(a) \\
= & \sum_{a=1}^{q-1} a \chi(a)-\chi(4) q \sum_{a=1}^{(q-1) / 2} \chi(a)-2 \chi(4) q \sum_{a=1}^{(q-1) / 4} \chi(a),
\end{aligned}
$$

where we have used the fact that $\chi(-1)=-1$ and

$$
\sum_{a=(q+3) / 4}^{(2 q-2) / 4} \chi(a)=-\sum_{a=(2 q+2) / 4}^{(3 q-3) / 4} \chi(a) .
$$

Now, from (3) and Lemma 3, we get

$4 \chi(4) \sum_{a=1}^{q-1} a \chi(a)=\sum_{a=1}^{q-1} a \chi(a)-(\chi(2)-2 \chi(4)) \sum_{a=1}^{q-1} a \chi(a)-2 \chi(4) q \sum_{a=1}^{(q-1) / 4} \chi(a)$.

That is,

$$
\sum_{a=1}^{(q-1) / 4} \chi(a)=\frac{\bar{\chi}(4)-\bar{\chi}(2)-2}{2 q} \sum_{a=1}^{q-1} a \chi(a)=\frac{\bar{\chi}(4)-\bar{\chi}(2)-2}{2 q} \sum_{a=1}^{q} a \chi(a) .
$$

Then from Lemma 1, we have

$$
\sum_{a=1}^{(q-1) / 4} \chi(a)=\frac{2+\bar{\chi}(2)-\bar{\chi}(4)}{2 i \pi} \tau(\chi) L(1, \bar{\chi})
$$

This is the assertion of Lemma 4 in the case of $q \equiv 1(\bmod 4)$. By the same method, we can also prove

$$
\sum_{a=1}^{(q-3) / 4} \chi(a)=\frac{2+\bar{\chi}(2)-\bar{\chi}(4)}{2 i \pi} \tau(\chi) L(1, \bar{\chi})
$$

if $q \equiv 3(\bmod 4)$. This completes the proof of Lemma 4 . 
Lemma 5. Let $q$ be any positive integer. Then for any positive integer $r$ with $(r, q)=1$, we have the identity

$$
\bar{B}_{n}\left(\frac{r}{q}\right)=-\frac{2 n !}{(2 i \pi)^{n}} q^{-n} \sum_{d \mid q} \frac{d^{n}}{\phi(d)} \sum_{\substack{\chi \bmod d \\ \chi(-1)=(-1)^{n}}} G(r, \bar{\chi}) L(n, \chi),
$$

where $G(r, \bar{\chi})=\sum_{a=1}^{d} \bar{\chi}(a) e(r a / d)$ is the Gauss sum.

Proof. From Theorem 12.19 of [1], we know that

$$
B_{n}(x)=-\frac{n !}{(2 i \pi)^{n}} \sum_{\substack{t=-\infty \\ t \neq 0}}^{+\infty} \frac{e(t x)}{t^{n}}
$$

if $0<x \leq 1$. So we can write

$$
\begin{aligned}
\bar{B}_{n}\left(\frac{r}{q}\right) & =-\frac{n !}{(2 i \pi)^{n}} \sum_{d \mid q} \sum_{\substack{t=-\infty \\
t \neq 0 \\
(t, d)=1}}^{+\infty} \frac{e\left(\frac{r \frac{q}{d} t}{q}\right)}{\left(t \frac{q}{d}\right)^{n}} \\
& =-\frac{n !}{(2 i \pi)^{n}} \sum_{d \mid q}\left(\frac{d}{q}\right)^{n} \sum_{\substack{t=-\infty \\
t \neq 0 \\
(t, d)=1}}^{+\infty} \frac{e(r t / d)}{t^{n}} .
\end{aligned}
$$

Now from the orthogonality relation for Dirichlet characters $\chi \bmod d$ we immediately get

$$
\begin{aligned}
\sum_{d \mid q}\left(\frac{d}{q}\right)^{n} \sum_{\substack{t=-\infty \\
t \neq 0 \\
t, d)=1}}^{+\infty} \frac{e(r t / d)}{t^{n}} & =q^{-n} \sum_{d \mid q} \frac{d^{n}}{\phi(d)} \sum_{\substack{t=-\infty \\
t \neq 0}}^{+\infty} \sum_{b=1}^{d} \sum_{\chi \bmod d} \frac{\chi(t \bar{b}) e(r b / d)}{t^{n}} \\
& =2 q^{-n} \sum_{d \mid q} \frac{d^{n}}{\phi(d)} \sum_{\substack{\chi \bmod d \\
\chi(-1)=(-1)^{n}}} G(r, \bar{\chi}) L(n, \chi),
\end{aligned}
$$

and the assertion follows.

3. Proof of the theorems. In this section, we complete the proofs of the theorems. Let $q=p>4$ be a prime. Noting that $G(r, \bar{\chi})=\chi(r) \tau(\bar{\chi})$ if $\chi$ is a primitive character, from Lemma 5 we have

$$
\bar{B}_{n}\left(\frac{r}{p}\right)=-\frac{2 n !}{(2 i \pi)^{n}} p^{-n} \sum_{d \mid p} \frac{d^{n}}{\phi(d)} \sum_{\substack{\chi \bmod d \\ \chi(-1)=(-1)^{n}}} \chi(r) \tau(\bar{\chi}) L(n, \chi)
$$




$$
= \begin{cases}-\frac{2 n !}{(2 i \pi)^{n}} p^{-n}\left(\zeta(n)+\frac{p^{n}}{\phi(p)} \sum_{\substack{\chi \bmod p \\ \chi(-1)=1}} \chi(r) \tau(\bar{\chi}) L(n, \chi)\right) & \text { if } n \text { is even, } \\ -\frac{2 n !}{(2 i \pi)^{n} \phi(p)} \sum_{\substack{\chi \bmod p \\ \chi(-1)=-1}} \chi(r) \tau(\bar{\chi}) L(n, \chi) & \text { if } n \text { is odd. }\end{cases}
$$
write

First, we prove Theorem 1 . If $n$ is even, from (6) and Lemma 2 we can

$$
\begin{aligned}
& \sum_{r \leq[p / 4]} \bar{B}_{n}\left(\frac{r}{p}\right) \\
= & -\frac{2 n !}{(2 i \pi)^{n}} p^{-n} \\
& \times\left(\zeta(n)\left[\frac{p}{4}\right]-\frac{i p^{n}}{2 \pi \phi(p)} \sum_{\substack{\chi \bmod p \\
\chi(-1)=1}} \bar{\chi}(4) \tau\left(\chi \chi_{4}\right) \tau(\bar{\chi}) L\left(1, \bar{\chi} \chi_{4}\right) L(n, \chi)\right)
\end{aligned}
$$

Noting that

$$
\begin{aligned}
\tau\left(\chi \chi_{4}\right) & =\sum_{a=1}^{4 p} \chi_{4}(a) e\left(\frac{a}{4 p}\right) \\
& =\sum_{a=1}^{4} \sum_{b=1}^{p} \chi(4 b+p a) \chi_{4}(4 b+p a) e\left(\frac{4 b+p a}{4 p}\right) \\
& =\sum_{a=1}^{4} \sum_{b=1}^{p} \chi(4 b) \chi_{4}(p a) e\left(\frac{b}{p}+\frac{a}{4}\right) \\
& =\chi(4) \chi_{4}(p)\left(\sum_{a=1}^{3} \chi_{4}(a) e\left(\frac{a}{4}\right)\right)\left(\sum_{b=1}^{p-1} \chi(b) e\left(\frac{b}{p}\right)\right) \\
& =\chi(4) \chi_{4}(p)\left(e\left(\frac{1}{4}\right)-e\left(\frac{3}{4}\right)\right)\left(\sum_{b=1}^{p-1} \chi(b) e\left(\frac{b}{p}\right)\right) \\
& =2 i \chi(4) \chi_{4}(p) \tau(\chi)
\end{aligned}
$$

and $\tau(\chi) \bar{\tau}(\chi)=p$ if $\chi(-1)=1$, we have

$$
\begin{aligned}
& \sum_{\substack{\chi \bmod p \\
\chi(-1)=1}} \bar{\chi}(4) \tau\left(\chi \chi_{4}\right) \tau(\bar{\chi}) L\left(1, \bar{\chi} \chi_{4}\right) L(n, \chi) \\
& =2 i \chi_{4}(p) p \sum_{\substack{\chi \bmod p \\
\chi(-1)=1}} L\left(1, \bar{\chi} \chi_{4}\right) L(n, \chi) .
\end{aligned}
$$


Now combining this with (7) we get

$$
\begin{aligned}
-\frac{2 n ! \chi_{4}(p) p}{(2 i)^{n} \pi^{n+1} \phi(p)} \sum_{\substack{\chi \bmod p \\
\chi(-1)=1}} L\left(1, \bar{\chi} \chi_{4}\right) L(n, \chi) \\
=\frac{2 n ! \zeta(n)}{(2 i \pi)^{n}} p^{-n}\left[\frac{p}{4}\right]+\sum_{r \leq[p / 4]} \bar{B}_{n}\left(\frac{r}{p}\right) .
\end{aligned}
$$

If $n$ is odd, similarly, from (6) and Lemma 4 we have

$$
\sum_{r \leq[p / 4]} \bar{B}_{n}\left(\frac{r}{p}\right)
$$

$$
\begin{aligned}
& =-\frac{2 n !}{(2 i \pi)^{n} \phi(p)} \sum_{\substack{\chi \bmod p \\
\chi(-1)=-1}} \frac{2+\bar{\chi}(2)-\bar{\chi}(4)}{2 i \pi} \tau(\chi) L(1, \bar{\chi}) \tau(\bar{\chi}) L(n, \chi) \\
& =\frac{2 n ! p}{(2 i \pi)^{n+1} \phi(p)} \sum_{\substack{\chi \bmod p \\
\chi(-1)=-1}}(2+\bar{\chi}(2)-\bar{\chi}(4)) L(1, \bar{\chi}) L(n, \chi) .
\end{aligned}
$$

That is,

$$
\sum_{\substack{\chi \bmod p \\ \chi(-1)=-1}}(2+\bar{\chi}(2)-\bar{\chi}(4)) L(1, \bar{\chi}) L(n, \chi)=\frac{(2 i \pi)^{n+1} \phi(p)}{2 n ! p} \sum_{r \leq[p / 4]} \bar{B}_{n}\left(\frac{r}{p}\right),
$$

where we have used the fact that $\tau(\chi) \tau(\bar{\chi})=-p$ if $\chi(-1)=-1$.

Now we prove Theorem 2 . From Lemmas 3 and 1, we easily get

$$
\sum_{a=1}^{[p / 2]} \chi(a)=\frac{(\bar{\chi}(2)-2) i}{\pi} \tau(\chi) L(1, \bar{\chi}) .
$$

Now by using the same method as in proving Theorem 1, we also obtain

$$
\begin{aligned}
-\frac{2 i n ! p}{(2 i)^{n} \pi^{n+1} \phi(p)} \sum_{\substack{\chi \bmod p \\
\chi(-1)=1}}(\bar{\chi}(2)-2) L(1, \bar{\chi}) L(n, \chi) & \\
& =\frac{n ! \zeta(n)(p-1)}{(2 i \pi p)^{n}}+\sum_{r \leq(p-1) / 2} \bar{B}_{n}\left(\frac{r}{p}\right)
\end{aligned}
$$

if $n$ is even, and

$$
\sum_{\substack{\chi \bmod p \\ \chi(-1)=-1}}(\bar{\chi}(2)-2) L(1, \bar{\chi}) L(n, \chi)=\frac{(2 i)^{n} \pi^{n+1} \phi(p)}{2 i n ! p} \sum_{r \leq(p-1) / 2} \bar{B}_{n}\left(\frac{r}{p}\right)
$$

if $n$ is odd. This completes the proof of the theorems. 
Acknowledgments. The authors express their gratitude to the referee for very helpful and detailed comments.

\section{References}

[1] T. M. Apostol, Introduction to Analytic Number Theory, Springer, New York, 1976.

[2] H. N. Liu and W. P. Zhang, On the mean value of $L(m, \chi) L(n, \bar{\chi})$ at positive integers $m, n \geq 1$, Acta Arith. 122 (2006), 51-56.

[3] H. Walum, An exact formula for an average of L-series, Illinois J. Math. 26 (1982), $1-3$.

[4] Z. F. Xu and W. P. Zhang, On the 2kth power mean of the character sums over short intervals, Acta Arith. 121 (2006), 149-160.

[5] W. P. Zhang, On the mean values of Dedekind sums, J. Théor. Nombres Bordeaux 8 (1996), 429-442.

[6] - On the general Dedekind sums and one kind identities of Dirichlet L-functions, Acta Math. Sinica 44 (2001), 269-272 (in Chinese).

Department of Mathematics

Northwest University

Xi'an, Shaanxi, P.R. China

E-mail: zfxu@nwu.edu.cn

wpzhang@nwu.edu.cn

Received on 15.11.2006

and in revised form on 2.8.2007 